\title{
Atividades antioxidante e fungitóxica do óleo essencial de capim-limão e do citral ${ }^{1}$
}

\author{
Antioxidant and fungitoxic activities of the lemongrass essential oil and citral
}

\author{
Luiz Gustavo de Lima Guimarães ${ }^{2 *}$, Maria das Graças Cardoso ${ }^{3}$, Paulo Estevão de Sousa ${ }^{4}$, Juliana de Andrade ${ }^{5}$ \\ e Sara Silveira Vieira ${ }^{5}$
}

\begin{abstract}
Resumo - Tendo em vista a importância dos óleos essenciais, objetivou-se, com este trabalho, avaliar a atividade antioxidante e os efeitos fungitóxicos do óleo essencial de capim-limão e do seu constituinte majoritário citral sobre a inibição micelial dos fitopatógenos Fusarium oxysporum cubense, Colletotrichum gloeosporioides, Bipolaris sp. e Alternaria alternata. A atividade antioxidante foi realizada, empregando os métodos de redução do radical livre DPPH e o ensaio de oxidação do sistema $\beta$ caroteno/ácido linoléico. Para cada metodologia, utilizou-se o delineamento inteiramente casualizado em esquema fatorial de 4 x 5, sendo quatro compostos (óleo essencial, citral, timol e $\alpha$-tocoferol) e cinco concentrações $\left(5 ; 10 ; 25 ; 50\right.$ e $\left.100 \mu \mathrm{g} \mathrm{L}^{-1}\right)$, com três repetições Os ensaios para verificação das atividades fungitóxicas foram realizados aplicando o teste de fumigação in vitro sobre a inibição micelial dos fungos fitopatogênicos estudados. Os tratamentos foram dispostos de forma inteiramente casualizada, com três repetições e em esquema fatorial com arranjo variável entre os fitopatógenos. O óleo essencial e o citral apresentaram pequena atividade antioxidante perante a metodologia que avalia a redução do radical estável DPPH, no entanto, perante a metodologia que emprega o sistema emulsificado $\beta$-caroteno/ácido linoléico estes compostos apresentaram atividades antioxidantes significativas. Nas atividades fungitóxicas, o óleo essencial e o citral apresentaram uma maior atividade sobre o fitopatógeno Alternaria alternata. Em relação aos valores de $\mathrm{IC}_{50}$ os menores valores apresentados pelo óleo essencial $\left(75,83 \mu \mathrm{g} \mathrm{mL}^{-1}\right)$ e pelo citral $\left(58,24 \mu \mathrm{g} \mathrm{mL}^{-1}\right)$ foram sobre o Bipolaris sp. O citral causou as maiores inibições miceliais, para todos os fitopatógenos.
\end{abstract}

Palavras-chave - Cymbopogon citratus. Fitopatógenos. Atividade Biológica. Metabólitos secundários.

\begin{abstract}
Given the importance of essential oils, this study evaluated the antioxidant activity and fungitoxic effects of lemongrass essential oil and its main constituent citral on mycelial inhibition of phytopathogens Fusarium oxysporum cubense, Colletotrichum gloeosporioides, Bipolaris sp. and Alternaria alternata. The antioxidant activity was evaluated by employing the DPPH free radical reduction method and oxidation tests of the system $\beta$-carotene/linoleic acid. For each method, a completely randomized design in a 4 x 5 factorial arrangement was used with four compounds (essential oil, citral, thymol and $\alpha$-tocopherol) and five concentrations $\left(5 ; 10 ; 25 ; 50\right.$ and $\left.100 \mathrm{Mg} \mathrm{L}^{-1}\right)$, with three replicates. The tests to determine fungitoxic activities were performed by applying the in vitro fumigation test on mycelial inhibition of studied phytopathogens. The treatments were arranged in a completely randomized design with three replicates and a factorial arrangement. The essential oil and citral showed little antioxidant activity by the methodology that measures the reduction of the stable radical DPPH; however, by the methodology that employs the emulsified $\beta$-carotene/linoleic acid system, these compounds showed significant antioxidant activities. At fungitoxic activities, the essential oil and citral showed greater activity against the phytopathogen Alternaria alternata. When compared to the values of $\mathrm{IC}_{50}$, the lowest values presented by the essential $\left(75.83 \mu \mathrm{g} \mathrm{mL}^{-1}\right)$ and citral $\left(58.24 \mu \mathrm{g} \mathrm{mL}^{-1}\right)$ oils were found for Bipolaris sp. Citral caused the greatest mycelial inhibition on all phytopathogens.
\end{abstract}

Key words - Cymbopogon citratus. Phytopathogens. Biological Activity. Secondary metabolites.

\footnotetext{
*Autor para correspondência

${ }^{1}$ Recebido para publicação em 01/09/2010; aprovado em 14/03/2011

Extraído do trabalho de pesquisa desenvolvida pelos Departamentos de Química e de Fitopatologia da Universidade Federal de Lavras

${ }^{2}$ Departamento de Química Ambiental, Campus Universitário de Gurupi/UFT, Gurupi-TO, Brasil, 77.402-970, 1gguimaraes@uft.edu.br

${ }^{3}$ Departamento de Química/UFLA, Lavras-MG, Brasil, 37.200-000, mcardoso@dqi.ufla.br,

${ }^{4}$ Departamento de Fitopatologia/UFLA, Lavras-MG, Brasil, 37.200-000, pauleste@ufla.br

${ }^{5}$ Programa de Pós-Graduação em Agroquímica/UFLA, Lavras-MG, Brasil, 37.200-000, juandrade_quimica@yahoo.com.br, saraufla@yahoo.com.br
} 


\section{Introdução}

Os óleos essenciais, constituintes voláteis orgânicos responsáveis pela fragrância de muitas plantas, são compostos que têm apresentado grande importância em determinadas pesquisas, por serem potencialmente úteis no controle fitossanitário, propiciando o desenvolvimento de técnicas que procuram diminuir os efeitos negativos de oxidantes, radicais e microrganismos que causam prejuízos nas indústrias alimentícias e na agricultura (BAKKALI et al., 2008).

O capim-limão (Cymbopogon citratus (D. C.) Stapf), pertencente à família das Poaceae, é uma planta aromática cultivada para produção comercial de óleo essencial, o qual geralmente apresenta como constituintes majoritários os monoterpenos citral (mistura isomérica de neral e geranial) e o mirceno. É largamente empregado como aromatizante em perfumaria e cosmética, na preparação de colônias, sabonetes e desodorantes. Porém, seu maior emprego tem sido na indústria farmacêutica, servindo de material de partida para síntese de importantes compostos como iononas, metil-iononas e vitamina A (PRINS et al., 2008).

Estudos tais como de Vardar-Unlus et al. (2003) demonstraram a atividade antioxidante do óleo essencial de Thymus pectinatus Fesch. Et Mey, de Morais et al. (2006) que encontraram para os óleos essenciais de algumas espécies de Croton do nordeste do Brasil atividades maiores que dos antioxidantes BHT e $\alpha-$ tocoferol, e de Eminagaoglu et al. (2007), apresentando atividades antioxidantes para os óleos essenciais de Satureja spicigera C. Koch Boiss e Satureja cuneifolia Ten maiores que aquelas apresentadas pelos compostos antioxidantes BHT e ácido ascórbico, têm demonstrado as propriedades antioxidantes de diversas plantas aromáticas no retardamento dos processos de peroxidação lípidica em alimentos e na neutralização de radicais livres.Muitos trabalhos relatam a atividade biológica dos óleos essenciais sobre diversos microrganismos, incluído fitopatógenos. Trabalhos de Cakir et al. (2005) constataram efeito fungicida do óleo essencial de Hypericum linarioides Bosse em seis espécies do gênero Fusarium ( $F$. acuminatum, $F$. culmorum, $F$. equiseti, $F$. oxysporum, $F$. sambucinum e $F$. solani). Bajpai et al. (2007), estudando o efeito fungitóxico do óleo essencial de Metasequoia glyptostroboides Miki ex $\mathrm{Hu}$, sobre fungos pertencentes ao gênero Fusarium e outros, observaram que esse óleo essencial na concentração de $1000 \mu \mathrm{g} \mathrm{mL}^{-1}$, causou uma inibição micelial de $63 \%$ sobre Fusarium oxysporum e Colletotricum capsici, 70\% sobre Phytophthora capsici, 56\% sobre Botrytis cinerea e Sclerotiorum sclerotiorum e 68\% sobre Fusarium solani, demonstrando, assim, a atividade fungitóxica desse óleo. A atividade nematostática e nematicida dos óleos essenciais de alecrim pimenta e capim citronela sobre Meloidogyne incognita foi demonstrada por Moreira et al. (2009).
Tendo em vista a importância do óleo essencial de capim-limão e os crescentes estudos sobre as atividades antioxidantes e fungitóxicas dos óleos essenciais, este trabalho objetivou avaliar a atividade antioxidante e os efeitos fungitóxicos do óleo essencial de capim-limão e do seu constituinte majoritário citral sobre o crescimento e/ou inibição micelial dos fitopatógenos Fusarium oxysporum cubense, Colletotrichum gloeosporioides, Bipolaris sp. e Alternaria alternata.

\section{Material e métodos}

\section{Óleo essencial}

O material vegetal (folhas frescas de capim-limão) foi coletado na Empresa de Produção de Sementes de Hortaliças "HORTIAGRO" localizada no município de Ijaci - MG. O método empregado para a extração do óleo essencial foi o de arraste a vapor, utilizando-se o aparelho de Clevenger modificado (GUIMARÃES et al., 2008).

\section{Análises cromatográficas}

A análise qualitativa do óleo essencial foi feita em cromatografia gasosa acoplada à espectrometria de massa CG/EM. O cromatógrafo utilizado foi o modelo Shimadzu CG-17A equipado com detector seletivo de massa modelo QP 5000. O equipamento foi operado nas seguintes condições: coluna capilar de sílica fundida com fase ligada DB5; temperatura do injetor de $220{ }^{\circ} \mathrm{C}$; programação da coluna com temperatura inicial de $40{ }^{\circ} \mathrm{C}$, sendo acrescidos $3{ }^{\circ} \mathrm{C}$ a cada minuto até atingir $240{ }^{\circ} \mathrm{C}$; gás carreador hélio; pressão inicial na coluna de 100,2 KPa; taxa de split 1:10 e volume injetado de $1 \mu \mathrm{L}$ ( $1 \%$ de solução em diclorometano). Foi injetada nas mesmas condições da amostra, uma série de padrões de hidrocarbonetos $\left(\mathrm{C}_{9} \mathrm{H}_{20} . . \mathrm{C}_{26} \mathrm{H}_{54}\right)$ (MJOS et al., 2006). Os espectros obtidos foram comparados com o banco de dados da biblioteca Wiley 229 e o índice Kovats, calculado para cada constituinte, foi comparado com o tabelado de acordo com Adams (2007).

Para a avaliação dos teores dos constituintes do óleo essencial utilizou-se um cromatógrafo gasoso Shimadzu GC 17A equipado com detector por ionização de chamas (DIC), nas mesmas condições operacionais descritas anteriormente, mantendo a pressão na coluna igual a $115 \mathrm{KPa}$ e utilizando o nitrogênio como gás de arraste. A quantificação de cada constituinte foi obtida por meio de normalização de áreas (\%).

Com a finalidade de corrigir as diferenças nas respostas apresentadas pelo detector DIC frente aos diferentes compostos, a quantificação dos constituintes majoritários citral (neral + geranial) e mirceno foi feita também pelo método da adição-padrão, utilizando padrões 
de citral (Aldrich, 95\% de pureza) e de mirceno (Acrõs, $90 \%$ de pureza). Para tal, foram feitas curvas analíticas das concentrações versus as respostas do detector (área do pico) para os compostos a serem quantificados. As soluções dos compostos (em hexano) foram preparadas nas seguintes concentrações: 4,$0 ; 6,0 ; 8,0 ; 10,0$ e 12,0 $\mathrm{mg} \mathrm{mL} \mathrm{m}^{-1}$ para o citral; 0,$5 ; 1,0 ; 1,5 ; 2,0$ e $2,5 \mathrm{mg} \mathrm{mL}^{-1}$ para o mirceno; para o óleo essencial foi utilizada uma concentração fixa de $10 \mathrm{mg} \mathrm{mL}^{-1}$, sendo os cálculos para determinação dos teores de citral e mirceno realizados de acordo com Harris (2008). As análises cromatográficas foram realizadas nas mesmas condições anteriores, sendo realizadas em triplicatas.

\section{Atividades biológicas}

As atividades biológicas estudadas basearam-se em atividade antioxidante do óleo essencial de capim-limão e do citral, e em atividade microbiológica dos vapores destes mesmos compostos e do mirceno. A atividade antioxidante foi realizada, empregando os métodos de redução do radical livre DPPH (2,2-difenil-1-picrilhidrazil) e o ensaio de oxidação do sistema $\beta$-caroteno/ácido linoléico, sendo utilizados como controle positivo os compostos timol e $\alpha$-tocoferol. As atividades fungitóxicas foram realizadas aplicando o teste fumigação in vitro sobre o crescimento/ inibição micelial dos fitopatógenos Alternaria alternata, Colletotrichum gloesporioides, Fusarium oxysporum cubense e Bipolaris sp. (TULLIO et al., 2006).

\section{Redução do DPPH}

A avaliação da atividade antioxidante diante do consumo de DPPH foi realizada de acordo com as metodologias de Sousa et al. (2007) e Lopes-Lutz et al. (2008) seguidas de pequenas modificações. Preparou-se uma solução de DPPH em metanol na concentração de $40 \mu \mathrm{g} \mathrm{mL}^{-1}$, a qual foi mantida sob refrigeração e ao abrigo da luz, e soluções do óleo essencial, do citral, do timol e do $\alpha$-tocoferol nas concentrações de 50;150;250; 500 e $1.000 \mu \mathrm{g} \mathrm{mL}^{-1}$, também em metanol. As misturas reacionais foram preparadas em tubos de ensaio, adicionando-se $0,3 \mathrm{~mL}$ das soluções das amostras a $2,7 \mathrm{~mL}$ da solução de DPPH em metanol. Sendo suas leituras de absorbância feitas em espectrofotômetro Shimadizu UV-1601PC, a 517 nm, no tempo de 60 min após junção das duas soluções. A porcentagem da atividade antioxidante foi calculada de acordo com a seguinte equação: [1-( $\left.\left.\mathrm{A}_{\text {amostra/ }} / \mathrm{A}_{\text {controle }}\right)\right]$ x 100, onde $\mathrm{A}_{\text {amostra é a absorbância da solução contendo todos }}$ os reagentes e Acontrole é a absorbância da solução controle (solução contendo todos os reagentes menos os compostos avaliados). Como branco foi utilizada a mistura de $2,7 \mathrm{~mL}$ de metanol e a solução metanólica dos compostos avaliados.

\section{Oxidação do sistema $\beta$-caroteno/ácido linoleico}

A avaliação da atividade antioxidante utilizando o ensaio do clareamento do $\beta$-caroteno foi realizada de acordo com as metodologias apresentadas por Wang et al. (2008) e Lopes-Lutz et al. (2008) com pequenas modificações. Foi preparada uma solução de $\beta$-caroteno

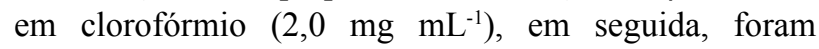
adicionados $60 \mu \mathrm{L}$ de ácido linoléico, $600 \mathrm{mg}$ de Tween $20 \AA \mathrm{e}$ $1,5 \mathrm{~mL}$ de clorofórmio, sendo o clorofórmio evaporado em rotaevaporador. Posteriormente, $150 \mathrm{~mL}$ de água destilada saturada com oxigênio foram acrescidos na mistura sob agitação constante (emulsão A). Em seguida 2,8 mL da emulsão A foram colocados em tubos de ensaio e sobre estas foram acrescidos $200 \mu \mathrm{L}$ das soluções metanólicas dos compostos em estudo nas concentrações apropriadas, para que os compostos avaliados estivessem presentes nas concentrações de $5 ; 10 ; 25 ; 50 ; 100 \mu \mathrm{g} \mathrm{mL}^{-1}$ na solução final. Paralelamente, foram preparadas duas soluções, uma sem o antioxidante (controle) e outra com os mesmos reagentes da emulsão A, sem o $\beta$-caroteno (emulsão B - branco).

As leituras das amostras foram realizadas nos tempos 0 e 60 minutos após adicionado o antioxidante sobre a emulsão A em espectrofotômetro Shimadizu UV-1601PC, a $470 \eta \mathrm{m}$. A porcentagem de inibição (porcentagem de atividade antioxidante AA\%) foi calculada por meio da seguinte fórmula; $\mathrm{AA} \%=100 \mathrm{x}$ (DC-DA)/DC, em que AA é a atividade antioxidante; DC é o grau de degradação do controle $=[\ln (\mathrm{a} / \mathrm{b}) / 60] ; \mathrm{DA}=$ $[\ln (\mathrm{a} / \mathrm{b}) / 60]=$ grau de degradação na presença da amostra; $\mathrm{a}=$ absorbância no tempo $0 ; \mathrm{b}=$ absorbância final $(60$ minutos após a incubação).Para cada metodologia, utilizou-se o delineamento inteiramente casualizado em esquema fatorial de $4 \times 5$, sendo quatro compostos (óleo essencial, citral, timol e $\alpha$-tocoferol) e cinco concentrações (5; $10 ; 25 ; 50$ e $\left.100 \mu \mathrm{g} \mathrm{L}^{-1}\right)$, com três repetições. Foram feitas as análises de variância, para verificar o efeito das concentrações sobre a atividade antioxidante de cada composto. Utilizou-se o Teste de Scott-Knott (5\%) para comparar os efeitos médios. As análises foram realizadas utilizando o programa SISVAR (FERREIRA, 2000).

\section{Atividade fungitóxica}

A atividade fungitóxica foi realizada no Laboratório de Epidemiologia e Manejo do Departamento de Fitopatologia da Universidade Federal de Lavras. Os fitopatógenos foram obtidos na micoteca do Departamento de Fitopatologia e registrados com os seguintes números: Alternaria alternata $\mathrm{CML} \mathrm{n}^{\circ} 1606$; Colletotrichum gloesporioides CML no 1449; Fusarium oxysporum cubense CML n ${ }^{\circ} 1450 ;$ Bipolaris sp. CML n ${ }^{\circ}$ 1451. Utilizou-se o método bioanalítico in vitro, onde avaliaram-se o efeito dos vapores do óleo essencial de 
capim-limão e de seus constituintes majoritários (citral e mirceno) sobre o crescimento e/ou inibição micelial dos fitopatógenos estudados.

As concentrações de óleo essencial e citral foram expressas em $\mu \mathrm{g} \mathrm{mL}^{-1}$ de ar, sendo utilizadas concentrações entre 8,80 a 106,00 para Bipolaris sp.; 35,00 a 284,00 para Fusarium oxysporum cubense; 70,90 a 241,30 para Colletotrichum gloesporioides e 17,70 a 177,00 para Alternaria alternata. Já para o mirceno foram utilizadas as mesmas concentrações para todos os microrganismos estudados, 35,50; 142,00; 283,90; 425,90; 567,90 e 709,90 $\mu \mathrm{g} \mathrm{mL} \mathrm{m}^{-1}$ de ar. Foram determinadas a concentração mínima inibitória (CMI), definida como a concentração de cada composto avaliado capaz de apresentar efeito inibitório de $0,5 \%$ sobre o crescimento micelial do microrganismo estudado (INOUYE et al., 2001), e a concentração necessário para inibir $50 \%$ do crescimento micelial $\left(\mathrm{IC}_{50}\right)$, sendo ambas calculadas por meio da análise de regressão. Os ensaios foram realizados em placas de Petri de $9 \mathrm{~cm}$ de diâmetro e $1,5 \mathrm{~cm}$ de altura, perfazendo um volume interno de $95,43 \mathrm{~mL}$, sendo adicionados $25 \mathrm{~mL}$ de meio de cultura em cada placa, resultando em um espaço livre de 70,43 $\mathrm{mL}$ de ar. Os óleos essenciais foram diluídos em éter etílico, sendo aplicado 0,25 mL destas soluções em cada placa sobre um papel de filtro circular de $4,00 \mathrm{~cm}$ de diâmetro aderido à parte superior da placa de Petri, obtendo as respectivas concentrações em estudo.

Após a solidificação do meio de cultura adicionado nas placas de Petri, discos de $7 \mathrm{~mm}$ de diâmetros contendo micélios das culturas fúngicas foram cortados de uma cultura pura e colocados no centro das placas. Em seguida foram colocadas as respectivas soluções de óleo essencial e éter etílico sobre o disco de papel de filtro aderido na parte superior da placa. Após a evaporação do éter etílico, as placas foram vedadas com filme plástico e levadas para a câmara de crescimento, com temperatura de aproximadamente $25^{\circ} \mathrm{C}$. Paralelamente prepararam-se duas testemunhas; uma composta apenas do fitopatógeno e do meio de cultura (Testemunha absoluta) e outra onde foram colocados $0,25 \mathrm{~mL}$ de éter no disco de papel aderido à parte superior da placa de Petri (Testemunha relativa). As medidas do crescimento micelial das culturas dos respectivos fungos foram realizadas no décimo dia após a inoculação dos fitopatógenos. Para tal, foram traçadas duas retas perpendiculares pela placa de Petri passando pelo centro do disco de $9 \mathrm{~mm}$; as leituras foram realizadas pelas medidas do diâmetro de crescimento das colônias. A porcentagem de inibição do crescimento micelial foi calculada para cada dosagem em relação à testemunha absoluta (BALBI-PEÑA et al., 2006).

Os tratamentos foram dispostos de formainteiramente casualizada, com três repetições e em esquema fatorial com arranjo variável entre os fitopatógenos, uma vez que, a faixa de concentração utilizada do óleo essencial e do citral, foi pré-determinada de acordo com a sensibilidade dos mesmos a esses compostos, utilizou-se concentrações e números de concentrações diferentes para todos os fitopatógenos. Foi feita a análise de variância para verificação dos efeitos de tratamentos, utilizando-se a análise de regressão para avaliar a porcentagem de inibição do crescimento micelial dos fitopatógenos, em relação à concentração, utilizando o programa estatístico SISVAR (FERREIRA, 2000).

\section{Resultados e discussão}

Os compostos encontrados no óleo essencial de capim-limão, seus respectivos teores (expressos em \% de normalização de áreas) e os índices de Kovats estão apresentados na Tabela 1. Os teores de citral e de mirceno determinados por meio da adição de padrão foram de 65,45 e $12,05 \%$ ( $\mathrm{m} / \mathrm{m}$ de óleo essencial), respectivamente, valores estes diferentes dos encontrados por meio da normalização de áreas 69,31\% de citral (geranial + neral) e $23,77 \%$ de mirceno, evidenciando as diferentes respostas do detector DIC de acordo com a estrutura de cada composto.

Estes resultados estão de acordo com diversos autores, que afirmam a presença do citral como componente majoritário do óleo essencial de capim-limão (CIMANGA et al., 2002; MARTINS et al., 2002; COSTA et al., 2005). Barbosa et al. (2008) relatam uma variação no teor de citral nos óleos essenciais comercializados no Brasil de 40,7 a $75,4 \%$ e no de mirceno de 0,24 a $7,29 \%$, estando os teores de mirceno um pouco abaixo do valor encontrado no óleo essencial estudado.

Os resultados dos quadrados médios e dos coeficientes de variação das análises de variância das atividades antioxidantes apresentadas pelo óleo essencial de capim-limão, citral, timol e $\alpha$-tocoferol, perante as metodologias de oxidação do sistema $\beta$-caroteno/ácido linoleico e de redução do DPPH estão apresentados na Tabela 2. De acordo com os dados, verifica-se que os tratamentos mostraram-se significativos $(p \leq 0,01)$ para os efeitos principais e também para a interação dos mesmos. Verificando-se efeito significativo dos fatores compostos, concentração e também da interação desses fatores sobre as atividades antioxidantes apresentadas pelos compostos estudados, diante de cada ensaio realizado.

As atividades antioxidantes estão apresentadas na Tabela 3. Observou-se a existência da variação da atividade antioxidante, dependendo do composto e da concentração, para cada metodologia separadamente.

O óleo essencial e o citral apresentaram pequena atividade antioxidante perante a metodologia que avalia a redução do radical estável DPPH, este fato pode ser 
Tabela 1 - Constituintes do óleo essencial de capim-limão identificados por CG/EM e seus respectivos teores expressos em normalização de área (\%). Lavras-MG, UFLA, 2010

\begin{tabular}{ccc}
\hline IK calculado* & Constituintes & Teor $(\%)$ \\
\hline 991 & Mirceno & 23,77 \\
1040 & $($ Z)- $\beta$-ocimeno & 0,50 \\
1051 & (E)- $\beta$-ocimeno & 0,28 \\
1199 & Linalol & 0,82 \\
1239 & Neral & 31,89 \\
1245 & Geraniol & 1,10 \\
1269 & Geranial & 37,42 \\
1292 & 2-undecanona & 0,27 \\
1375 & Geranil acetato & 0,10 \\
1415 & E-Cariofileno & 0,10 \\
& Outros & 3,75 \\
\hline Total & & 100 \\
\hline
\end{tabular}

*Índice de Kovats calculado para os compostos

Tabela 2 - Resumo dos quadrados médios e dos coeficientes de variação (CV) da análise de variância das atividades antioxidantes apresentadas pelo óleo essencial de Capim limão, citral, timol e $\alpha$-tocoferol, para os dois ensaios utilizados. Lavras-MG, UFLA, 2010

\begin{tabular}{lccc}
\hline \multirow{2}{*}{ Causas de variação } & \multirow{2}{*}{ G. L. } & \multicolumn{2}{c}{ Quadrados médios } \\
\cline { 3 - 4 } & & $\beta$-caroteno/ácido linoleico & Redução do DPPH \\
\hline Compostos (A) & 3 & $8000,81^{*}$ & $19911,90^{*}$ \\
Concentrações (B) & 4 & $3383,95^{*}$ & $1245,23^{*}$ \\
A X B & 12 & $223,55^{*}$ & $510,29^{*}$ \\
Resíduo & 40 & 37,34 & 0,25 \\
\hline C. V. (\%) & - & 14,80 & 1,69 \\
\hline * & & &
\end{tabular}

* valor significativo $1 \%$ pelo teste $\mathrm{F}$

justificado devido a não facilidade de doação de um hidrogênio por parte destes compostos, para neutralizar o radical DPPH, uma vez que esta metodologia se baseia no descoramento de uma solução composta por radicais estáveis DPPH de cor violeta quando na presença de substâncias que podem ceder um átomo de hidrogênio, neutralizando assim o radical (BRAND-WILLIAMS et al., 1995). Por outro lado Sacchetti et al. (2005) avaliando as atividades antioxidantes de vários óleos essenciais, encontraram mais de $60 \%$ de atividade antioxidante para o óleo essencial de capim-limão, que apresentava um teor de $73,58 \%$ de citral em sua composição, avaliando a capacidade de neutralizar o radical estável DPPH; no entanto esta diferença pode ser justificada, uma vez, que na metodologia utilizada por tais autores o óleo essencial foi emulsificado em Tween 20 e a solução foi mantida em $\mathrm{pH}$ constante por um sistema tamponado de Tris- $\mathrm{HCl}(\mathrm{pH} \mathrm{7,4).}$
Perante a metodologia que emprega o sistema emulsificado $\beta$-caroteno/ácido linoléico estes compostos apresentaram atividades antioxidantes crescentes de acordo com as concentrações utilizadas, chegando a atividades de $46,45 \%$ e de $38,00 \%$ na concentração de $100 \mu \mathrm{g} \mathrm{L}^{-1}$ para o óleo essencial e seu constituinte majoritário (citral), respectivamente. No entanto em relação às atividades apresentadas pelo timol e o $\alpha$ tocoferol estas atividades ainda são baixas. De acordo com Kulisic et al. (2004) compostos que apresentam o grupo $\mathrm{CHO}$ em sua estrutura apresentam valores consideráveis de atividades antioxidantes diante o sistema $\beta$-caroteno/ ácido linoléico, uma vez que compostos com valores intermediários de polaridades são menos diluídos na fase aquosa da emulsão, estando assim em maior concentração juntos à fase lipídica, sendo desta maneira mais efetivos na proteção do ácido linoléico. 
Tabela 3 - Atividades antioxidantes dos compostos estudados diante as metodologias empregadas. Lavras-MG, UFLA, 2010

\begin{tabular}{lccccc}
\hline \multirow{2}{*}{ Metodologia } & & \multicolumn{4}{c}{ Compostos } \\
\cline { 3 - 6 } & & Óleo essencial & Citral & Timol & $\alpha$-Tocoferol \\
\hline & $5 \mu \mathrm{g} \mathrm{L}^{-1}$ & $0,56 \mathrm{aA}$ & $0,51 \mathrm{aA}$ & $20,73 \mathrm{bA}$ & $34,67 \mathrm{cA}$ \\
Sequestro de DPPH & $10 \mu \mathrm{g} \mathrm{L}^{-1}$ & $-0,11 \mathrm{aA}$ & $1,04 \mathrm{aA}$ & $27,26 \mathrm{bB}$ & $69,94 \mathrm{cA}$ \\
& $25 \mu \mathrm{g} \mathrm{L}^{-1}$ & $0,08 \mathrm{aA}$ & $1,10 \mathrm{bA}$ & $39,65 \mathrm{cC}$ & $93,13 \mathrm{dC}$ \\
& $50 \mu \mathrm{g} \mathrm{L}^{-1}$ & $0,70 \mathrm{aA}$ & $0,75 \mathrm{aA}$ & $49,91 \mathrm{bD}$ & $92,85 \mathrm{cC}$ \\
& $100 \mu \mathrm{g} \mathrm{L}^{-1}$ & $2,48 \mathrm{bB}$ & $0,71 \mathrm{aA}$ & $61,82 \mathrm{cE}$ & $92,39 \mathrm{dC}$ \\
\hline Sistema $\beta$-caroteno/ácido linoléico & $5 \mu \mathrm{g} \mathrm{L}^{-1}$ & $7,68 \mathrm{aA}$ & $5,69 \mathrm{aA}$ & $20,73 \mathrm{bA}$ & $34,67 \mathrm{cA}$ \\
& $10 \mu \mathrm{g} \mathrm{L}^{-1}$ & $8,70 \mathrm{aA}$ & $15,94 \mathrm{aB}$ & $27,26 \mathrm{bA}$ & $69,94 \mathrm{cB}$ \\
& $25 \mu \mathrm{g} \mathrm{L}^{-1}$ & $28,66 \mathrm{aB}$ & $39,26 \mathrm{bC}$ & $39,64 \mathrm{bB}$ & $92,88 \mathrm{cC}$ \\
& $50 \mu \mathrm{g} \mathrm{L}^{-1}$ & $33,14 \mathrm{aB}$ & $30,77 \mathrm{aC}$ & $49,91 \mathrm{bC}$ & $93,09 \mathrm{cD}$ \\
& $100 \mu \mathrm{g} \mathrm{L}^{-1}$ & $46,45 \mathrm{aC}$ & $38,00 \mathrm{aC}$ & $61,82 \mathrm{bD}$ & $81,28 \mathrm{cD}$ \\
\hline
\end{tabular}

* Médias seguidas pela mesma letra minúscula nas linhas e pela mesma letra maiúscula nas colunas não diferem significativamente pelo teste de Scott-Knott $(\mathrm{p} \leq 0,05)$

Os resultados da atividade fungitóxica do óleo essencial de capim-limão e do citral sobre o crescimento micelial dos fitopatógenos Fusarium oxysporum cubense, Colletotrichum gloesporioides, Bipolaris sp. e Alternaria alternata, estão apresentados na Figura 1.

A inibição micelial das quatro espécies fúngicas foi calculada em relação ao crescimento micelial da testemunha absoluta de cada espécie. O crescimento micelial da testemunha relativa de cada espécie não diferenciou significativamente de sua testemunha absoluta. Houve diferença significativa para as diferentes concentrações de óleo essencial e citral sobre a inibição do crescimento micelial dos microrganismos estudados. $\mathrm{O}$ composto mirceno não apresentou atividade fungitóxica, uma vez que, a sua inibição micelial foi igual a zero para todos os fitopatógenos.

De acordo com as análises estatísticas de regressão, as inibições dos crescimentos miceliais dos fungos diante das concentrações dos compostos estudados apresentaram um comportamento linear, exceto para a inibição micelial do Colletotrichum gloesporioides diante as concentrações de citral, a qual obedeceu a uma equação de terceira ordem.

As CMI e as $\mathrm{IC}_{50}$ calculadas por meio das análises de regressão estão apresentadas na Tabela 4. Devido a inibição do crescimento micelial do fitopatógeno Colletotrichum gloesporioides perante às diferentes concentrações de citral não obedecer uma equação de primeiro grau, não foi possível calcular a $\mathrm{IC}_{50}$ para o citral sobre o crescimento micelial deste fitopatógeno.

Observa-se que o fitopatógeno Alternaria alternata foi o mais sensível diante do óleo essencial de capim-limão e do citral, uma vez que os valores de CMI apresentados pelos compostos sobre este microrganismo foram os menores, já o Colletotrichum gloesporioides foi o menos sensível, pois sobre este foram apresentados os maiores valores de CMI. No entanto, ao se observar esta sensibilidade em relação aos valores de $\mathrm{IC}_{50}$, o microrganismo mais sensível perante os compostos foi o Bipolaris sp., pois sobre este, os valores apresentados por ambos os compostos foram os menores. O Fusarium oxysporum cubense foi o fitopatógeno mais resistente à ação destes compostos, uma vez que, sobre o mesmo foram encontrados os maiores valores de $\mathrm{IC}_{50}$ para ambos os compostos. Diante disso observa-se que a maior ou menor sensibilidade dos microrganismos frente aos compostos estudados está relacionada a uma faixa restrita da concentração dos compostos, demonstrando que uma baixa CMI não implica em uma baixa $\mathrm{IC}_{50}$.

O citral foi o composto que apresentou os menores valores de $\mathrm{CMI}$ e $\mathrm{IC}_{50}$ para todos os fitopatógenos em relação ao óleo essencial, evidenciando a sua atividade fungitóxica e a sua importância na atividade do óleo essencial, uma vez que o também constituinte majoritário mirceno não apresentou atividade alguma sobre a inibição do crescimento micelial dos microrganismos estudados. Esses resultados corroboram com aqueles encontrados por Shukla et al. (2009), que demonstraram a atividade fungitóxica do óleo essencial de Lippia alba e de seus constituintes majoritários geranial e neral sobre diversos fitopatógenos, entre esses Alternata alternata que teve inibição de $100 \%$ do seu crescimento micelial pelo geranial e de 79,4\% pelo neral e Fusarium oxysporum que teve seu crescimento micelial inibido em $96,6 \%$ e $82,8 \%$ pelo geranial e neral, respectivamente. 


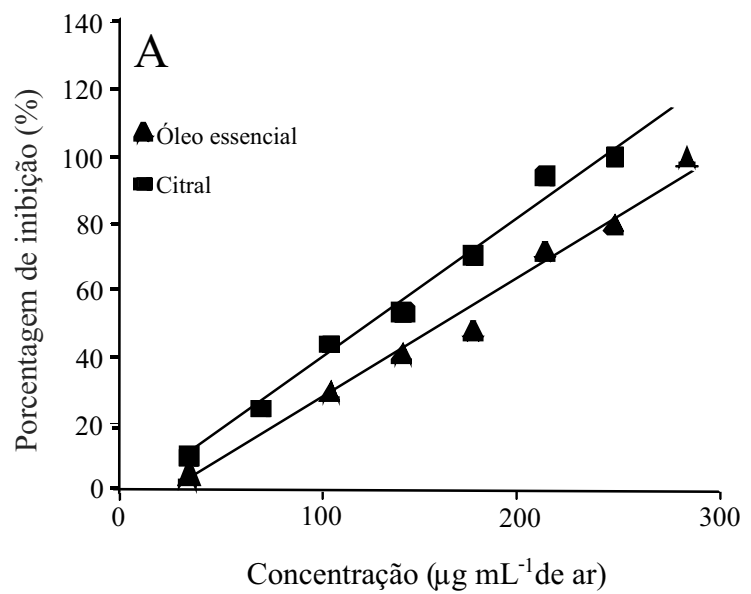

Citral: $\mathrm{y}=-5,6138+0,438^{*} \mathrm{x} \quad \mathrm{R}^{2}=0,9891$

Óleo esencial: $\mathrm{y}=-10,931+0,3757 * \mathrm{x} \quad \mathrm{R}^{2}=0,9852$

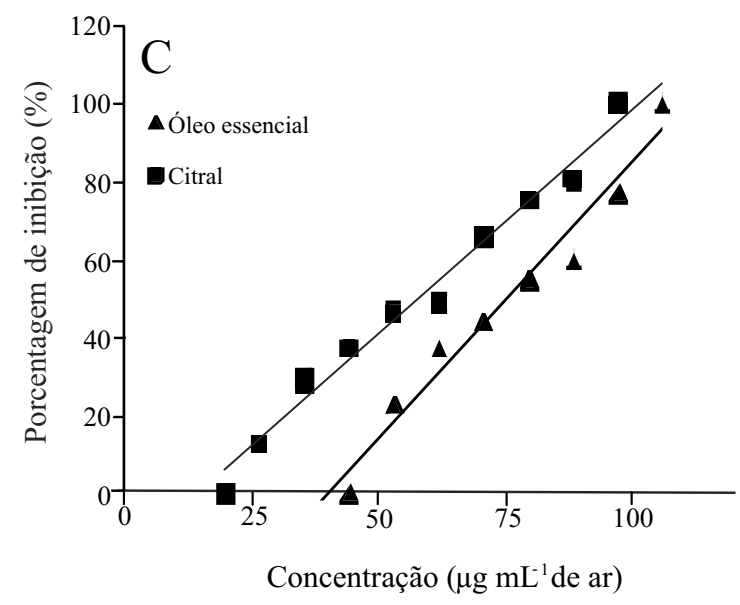

$\begin{array}{ll}\text { Citral: } \mathrm{y}=-18,203+1,171^{*} \mathrm{x} & \mathrm{R}^{2}=0,9833 \\ \text { Óleo essencial: } \mathrm{y}=-57,298+1,415^{*} \mathrm{x} & \mathrm{R}^{2}=0,9656\end{array}$

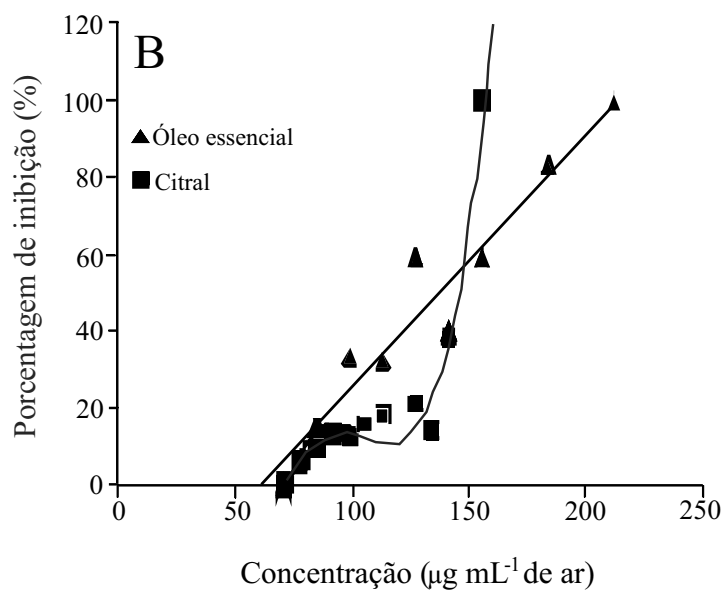

$\begin{array}{ll}\text { Óleo essencial: } y=-40,285+0,659 * x & R^{2}=0,9322 \\ \text { Citral: } y=-760,84+228,56 * x-221,983 * x^{2}+709,497 * x^{3} & R^{2}=0,9657\end{array}$

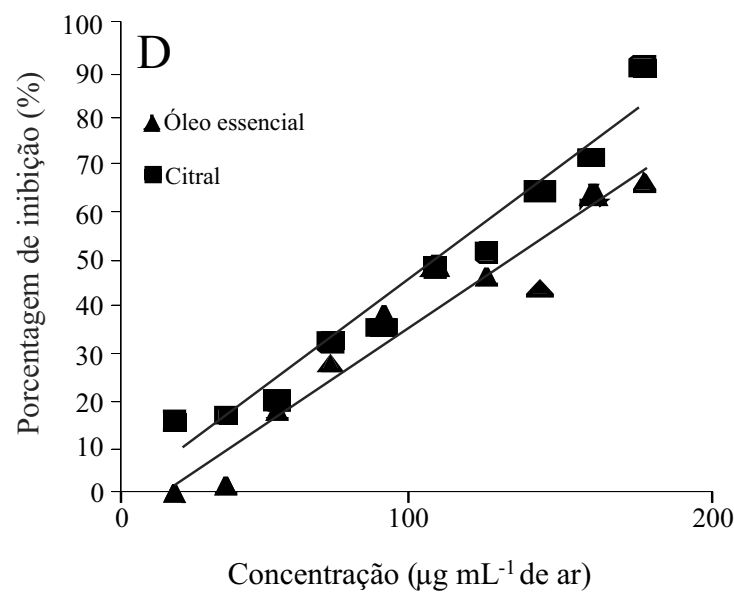

$\begin{array}{ll}\text { Citral: } y=-0,7447+0,463^{*} \mathrm{x} & \mathrm{R}^{2}=0,9605 \\ \text { Óleo essencial: } \mathrm{y}=-5,5598+0,418 * \mathrm{x} & \mathrm{R}^{2}=0,9357\end{array}$

*significativo a $1 \%$ de probabilidade

Figura 1 - Atividade fungitóxica do óleo essencial de capim-limão e do citral sobre o crescimento micelial dos fitopatógenos Fusarium oxysporum cubense (A), Colletotrichum gloesporioides (B), Bipolaris sp. (C) e Alternaria alternata (D)

As atividade fungitóxicas dos vapores do óleo essencial de capim-limão e do citral foram avaliadas sobre diversas espécies de Trichophyton por Inouye et al. (2001), os quais encontraram valores de CMI de 1 e $0,5 \mu \mathrm{g} \mathrm{mL}^{-1}$ de ar para o óleo essencial e o citral, respectivamente. Em comparação com a técnica de difusão em ágar estes mesmos autores demonstraram que por fumigação os CMI são menores, revelando uma maior atividade por meio dessa técnica, ainda segundo estes mesmos autores esta diferença se deve ao efeito combinado da ação dos vapores no micélio ou nos esporos antes da absorção dos compostos pelo ágar. Os vapores dos óleos essenciais de orégano, perilla, melaleuca, lavanda, cravo-da-índia e gerânio apresentaram CMI de $2 ; 2 ; 16 ; 16 ; 4 ; 8 \mu \mathrm{g} \mathrm{mL}^{-1}$, respectivamente, sobre Trichophyton mentogrophytis (INOUYE et al., 2006). Tzartzakis et al. (2007) avaliaram a atividade antifúngica do óleo essencial de capimlimão contra Colletotrichum coccodes, Botryts cinerea, Cladosporium herbarum, Rhizopus stolonifer e Aspergillus niger pelo teste de difusão em ágar, e observaram atividade contra a germinação de esporos, crescimento do tubo germinativo e micelial perante todos os fungos.

Silva et al. (2008) demonstraram a atividade antifúngica do óleo essencial de capim-limão e de seu constituinte majoritário citral sobre espécies de candida spp, quando empregados $8,0 \mu \mathrm{L}$ de cada composto, encontrando halos de inibição maiores que $40 \mathrm{~mm}$ de 
Tabela 4 - Valores das CMI e IC 50 $_{0}$ para o óleo essencial e seu constituinte majoritário (citral) perante a inibição do crescimento micelial dos fitopatógenos Fusarium oxysporum cubense, Colletotrichum gloesporioides, Bipolares spp e Alternaria alternata. Lavras-MG, UFLA, 2010

\begin{tabular}{lcccc}
\hline \multirow{2}{*}{ Fitopatógenos } & \multicolumn{2}{c}{ Óleo essencial } & \multicolumn{2}{c}{ Citral } \\
\cline { 2 - 5 } & $\mathrm{IC}_{50}\left(\mu \mathrm{g} \mathrm{mL} \mathrm{mL}^{-1}\right)$ & $\mathrm{CMI}\left(\mu \mathrm{g} \mathrm{mL}^{-1}\right)$ & $\left.\mathrm{IC}_{50}(\mu \mathrm{g} \mathrm{mL})^{-1}\right)$ & $\mathrm{CMI}\left(\mu \mathrm{g} \mathrm{mL}{ }^{-1}\right)$ \\
\hline Fusarium oxysporum cubense & 162,18 & 30,42 & 126,97 & 13,96 \\
Colletotrichum gloesporioides & 137,00 & 61,89 & - & 70,90 \\
Bipolaris sp. & 75,83 & 40,84 & 58,24 & 15,97 \\
Alternaria alternata & 132,92 & 14,49 & 109,60 & 2,9 \\
\hline
\end{tabular}

diâmetro, sendo a espécie $C$. albicans a mais sensível diante ao efeito fungitóxico do óleo essencial de capim-limão e do citral. Lee et al. (2008) observaram que os compostos citronelal, neral, geraniol e geranial na concentração de 28 x $10^{-3} \mathrm{mg} \mathrm{mL}^{-1}$ de ar foram capazes de inibir em $100 \%$ o crescimento micelial de Phytophthora cactorum.

Apesar de muitos óleos essenciais e de seus compostos apresentarem atividades biológicas sobre os mais diversos microrganismos, pouco se sabe a respeito de seus mecanismos de ação. Kurita et al. (1981) afirmam que a atividade fungicida do citral é decorrente da alta capacidade apresentada por este composto de receber elétrons provenientes da célula do fungo, por meio de uma transferência de carga com um elétron doador presente nesta célula, resultando assim na morte do fungo. Trabalhos recentes, como o de Kumar et al. (2008) relatam a importância da hidrofobicidade dos óleos essenciais e de seus constituintes, sendo assim capazes de interagirem com a camada lipídica das membranas celulares, causando alterações em suas estruturas e as tornando menos seletivas, podendo ocasionar o extravasamento de íons e outros constituintes celulares.

\section{Conclusões}

1. O óleo essencial de capim-limão e o seu constituinte majoritário citral apresentaram atividade antioxidante apenas no ensaio de oxidação do sistema $\beta$-caroteno/ ácido linoléico;

2. Todos os microrganismos utilizados nos ensaios fungitóxicos tiveram seu crescimento micelial inibido pelo óleo essencial de capim-limão e pelo citral;

3. Os menores valores de $\mathrm{CMI}$ e de $\mathrm{IC}_{50}$ em relação à inibição micelial de todos os fitopatógenos utilizados foram apresentados pelo citral.

\section{Agradecimentos}

Ao Departamento de Química da Universidade Federal de Viçosa (UFV), à Fundação de Apoio a Pesquisa e Extensão de Minas Gerais (FAPEMIG) e ao Conselho Nacional de Desenvolvimento Científico e Tecnológico (CNPq).

\section{Referências}

ADAMS, R. P. 2007. Identification of Essential Oil Components by Gas Chromatography/Mass Spectrometry. 4. ed. Carol Stream: Allured Publishing Corporation, 2007.800 p.

BAJPAI, V. K.; RAHMAN, A.; KANG, S. C. Chemical composition and anti-fungal properties of the essential oil and crude extracts of Metasequoia glyptostroboides Miki ex Hu. Industrial Crops and Products, v. 26, n. 01, p. 28-35, 2007.

BAKKALI, F. et al. Biological effects of essential oils: a review. Food and Chemical Toxicology, v. 46, n. 02, p. 446-475, 2008.

BALBI-PEÑA, M. I. et al. Controle de Alternaria solani em tomateiro por extratos de Curcuma longa e Curcumina - I. avaliação in vitro. Fitopatologia Brasileira, v. 31, n. 03, p. 310-314, 2006.

BARBOSA, L. C. A. et al. Evaluation of the chemical composition of Brazilian commercial Cymbopogon citratus (D. C.) Stapf samples. Molecules, v. 13, p. 1864-1874, 2008.

BRAND-WILLIAMS, W.; CUVELIER, M. E.; BERSET, C. Use of free radical method to evaluate antioxidant activity. LebensmittelWissenschaft und-Technologie, v. 28, p. 25-30, 1995.

CAKIR, A. et al. Antifungal properties of essential oil and crude extracts of Hypericum linarioides Bosse. Biochemical Systematics and Ecology, v. 33, p. 245-256, 2005.

CIMANGA, K. et al. Correlation between chemical composition and antibacterial activity of essential oils of some aromatic medicinal plants growing in the Democratic Republic of Congo. Journal of Ethnopharmacology, v. 79, n. 02, p. 213-220, 2002.

COSTA, L. C. do B. et al. Secagem e fragmentação da matéria seca no rendimento e composição do óleo essencial de capimlimão. Horticultura Brasileira, v. 23, n. 04, p. 956-959, 2005. 
EMINAGAOGLU, O. et al. The in vitro antioxidant properties of the essential oils and methanol extracts of Satureja spicigera (K. Koch) Boiss and Satureja cuneifolia ten. Food Chemistry, v. 100, p. 339-343, 2007.

FERREIRA, D. F. Análises estatísticas por meio do Sisvar para Windows versão 4.0. In: REUNIÃO ANUAL DA REGIÃO BRASILEIRA DA SOCIEDADE INTERNACIONAL DE BIOMETRIA, 45., 2000, São Carlos. Anais... São Carlos: UFSCar, 2000. p. 255-258.

GUIMARÃES, L. G. L. et al. Influência da luz e da temperatura sobre a oxidação do óleo essencial de capim-limão (Cymbopogon citratus (D.C.) STAPF). Química Nova, v. 31, n. 06, p. $1476-1480,2008$.

HARRIS, D. C. Análise Química Quantitativa. 7. ed. Rio de Janeiro: Técnicos e Científicos, 2008. 867 p.

INOUYE, S.; UCHIDA, K.; YAMAGUCHI, H. In vitro e in vivo anti-Trichophyton activity of essential oils by vapor contact. Mycoses, v. 44, p. 99-107, 2001.

INOUYE, S. et al. The vapor activity of oregano, perilla, tea tree, lavender, clove and geranium oils against a Trichophyton mentegrophytes in a closed box. Journal of Infection and Chemotherapy, v. 12, p. 349-354, 2006.

KULISIC, T. et al. Use of different methods for testing antioxidative activity of oregano essential oil. Food Chemistry, v. 85, p. 633-640, 2004.

KUMAR, A. et al. Assessment of Thymus vulgaris L. essential oil as a safe botanical preservative against post harvest fungal infestation of food commodities. Innovative Food Science and Emerging Technologies, v. 9, p. 575-580, 2008.

KURITA, N. et al. Antifungal activity of components of essential oils. Agricultural and Biological Chemistry, v. 45, p. 945-952, 1981.

LEE, Y. S. et al. Antifungal activity of Myrtaceae essential oils and their components against three phytopathogenic fungi. Flavour and Fragrance Journal, v. 23, p. 23-28, 2008.

LOPES-LUTZ, D. et al. Screening of chemical composition, antimicrobial and antioxidant activities of Artemisia essential oil. Phytochemistry, v. 69, p. 1732-1738, 2008.

MARTINS, P. M. et al. Influência da temperatura e velocidade do ar de secagem no teor e composição química do óleo essencial de capim-limão. Acta Horticulture, v. 569, p. 155-160, 2002.
MJOS, S. A.; MEIER,S.; BOITSOV, S. Alkylphenol retention indices. Journal of Chromatography, v. 1123, p. 98-105, 2006.

MORAIS, S. M. et al. Atividade antioxidante de óleos essenciais de espécies de Croton do nordeste do Brasil. Química Nova, v. 29, n. 5, p. 907-910, 2006.

MOREIRA, F. J. C.; SANTOS, C. D. G.; INNECO, R. Eclosão e mortalidade de juvenis $\mathrm{J} 2$ de Meloidogyne incognita raça 2 em óleos essenciais. Revista Ciência Agronômica, v. 40, n. 03, p. 441-448, 2009.

PRINS, C. L. et al. Efeitos de confinamento do sistema radicular sobre capim-limão (Cymbopogon citratus). Revista Ciência Agronômica, v. 39, n. 03, p. 416-421, 2008.

SACCHETTI, G. et al. Comparative evaluation of 11 essential oils of different origin as functional antioxidants, antiradicals and antimicrobials in foods. Food Chemistry, v. 91, p. 621-632, 2005.

SHUKLA, R. et al. Efficacy of Lippia alba (Mill) N. E. Brown essential oil and its monoterpenes aldehyde constituents aganst fungi isolated from some edible legume seeds and aflatoxin $\mathrm{B}_{1}$ production. International Journal of Food Microbiology, v. 135, p.165-170, 2009.

SILVA, C. B. et al. Antifungal activity of the lemongrass oil and citral against Candida spp. The Brazilian Journal of Infectios Diseases, v. 12, p. 1763-1766, 2008.

SOUSA, C. M. M. et al. Fenóis totais e atividade antioxidante de cinco plantas medicinais. Química Nova, v. 30, n. 2, p. 351355, 2007.

TULLIO, V. et al. Antifungal activity of essential oils against filamentous fungi determined by broth microdilution and vapour contact methods. Journal of Applied Microbiology, v. 102, p. 1544-1550, 2006.

TZARTZAKIS, N. G.; ECONOMAKIS, C. D. Antifungal activity of lemongrass (Cymbopogon citratus L.) essential oil against key postharvest pathogens. Innovative Food Science \& Emerging Technologies, v. 8, p. 253-258, 2007.

VARDAR-UNLUS, G. et al. Antimicrobial and antioxidant activity of the essential oil and methanol extracts of Thymus pectinatus Fesch. Et Mey. var. pectinatus (lamiaceae). Journal of Agricultural and Food Chemistry, v.51, p. 63-67, 2003.

WANG, W. et al. Antioxidative activity of Rosmarinus officinalis L. essential oil compared to its main components. Food Chemistry, v. 108, p. 1019-1022, 2008. 